\title{
Comportamento ingestivo de ovinos no período diurno em pastagem de azevém anual em diferentes estádios fenológicos
}

\section{Renato Borges de Medeiros ${ }^{1}$, Carlos Eduardo da Silva Pedroso ${ }^{2}$, João Batista Jornada da Jornada $^{2}$, Marcelo Abreu da Silva ${ }^{1}$, João Carlos de Saibro ${ }^{3}$}

\author{
${ }^{1}$ DPFA - Faculdade de Agronomia, UFRGS, Porto Alegre, RS, CEP: 91540-000. \\ ${ }^{2}$ Mestrando - DPFA, Fac. de Agronomia, UFRGS. \\ ${ }^{3}$ Colaborador Convidado, DPFA - Faculdade de Agronomia, UFRGS.
}

RESUMO - Este trabalho foi realizado com o objetivo de avaliar o comportamento ingestivo no período diurno de ovelhas Corriedale em final de gestação e início de lactação sob pastejo em uma área de 1,3 ha de azevém anual (Lolium multiflorum Lam.) nos estádios vegetativo, pré-florescimento e de florescimento. Foram determinados o tempo de pastejo, a taxa de bocado, o tamanho de bocado, o teor de PB da forragem e os consumos de MS e PB. Os maiores tempos de pastejo e de consumo de MS e PB, para os três estádios do azevém, foram observados ao final da tarde. Nos estádios vegetativo e pré-florescimento, os tempos de pastejo e os consumos de MS e PB registrados no início da manhã (7 às 7h50) foram semelhantes aos verificados no final da tarde. No estádio pré-florescimento, registraram-se ainda nestes horários de pico de pastejo as maiores taxas de bocado e peso de bocado, as quais, associadas ao maior tempo de pastejo, determinaram os maiores consumos de forragem ao longo do dia. Os menores tempos de pastejo ocorreram das 8 às 9h50 durante o estádio vegetativo; das 8 às 8 h50 e das 12 às $12 \mathrm{~h} 50$ durante o pré-florescimento; e das 7 às $8 \mathrm{~h} 50$ durante o florescimento. Conseqüentemente, nesses mesmos horários foram verificados os menores consumos de MS e PB para os três estádios fenológicos estudados.

Palavras-chave: comportamento diurno, consumo, Lolium multiflorum, pastejo seletivo

\section{Diurnal ingestive behavior of sheep grazing annual ryegrass at different phenological growth stages}

\begin{abstract}
The objective of this trial was to evaluate the diurnal ingestive behavior of late pregnant-early lactating Corriedale ewes grazing annual ryegrass (Lolium multiflorum Lam.) at different phenological growth stages (vegetative, preflowering and flowering). Animals were maintained in paddocks of 1.3 ha from July 21 to November 11 of 2000. Grazing time, bite rate, bite weight, crude protein (CP) content per bite, and forage dry matter (DM) and CP intakes were measured. The longest grazing time and the greatest forage DM and CP intakes were observed at late afternoon on all three phenological growth stages of annual ryegrass. Grazing time and intakes of CP and DM observed early in the morning (7:00 to 7:50 AM) during the vegetative and pre-flowering phenological growth stages were similar to those registered at late afternoon. In addition, the greater bite rates and bite weights observed at pre-flowering plus the longer grazing time at this growth stage resulted in the highest diurnal intake of forage DM. The shortest grazing times were found from 8:00 to 9:50 AM, from 8:00 to 8:50 $\mathrm{AM}$ and from 12:00 $\mathrm{AM}$ to 12:50 $\mathrm{PM}$, and from 7:00 to 8:50 $\mathrm{AM}$, respectively, for the vegetative, pre-flowering, and flowering phenological growth stages, which coincided with the lowest intakes of forage DM and CP.
\end{abstract}

Key Words: diurnal grazing behavior, forage intake, Lolium multiflorum, selective grazing

\section{Introdução}

No desenvolvimento dos estádios fenológicos do azevém anual, verifica-se crescente acúmulo de colmos e material senescente no resíduo da pastagem, determinando diminuição na qualidade da dieta (Pontes et al., 2003).

Diante da ascendente restrição nutricional, os animais desenvolvem diferentes estratégias de pastejo em adaptação à nova condição do ambiente de pastejo. Na literatura são relacionadas como principais estratégias utilizadas pelos animais variações no tempo de pastejo, na taxa de bocados, no peso do bocado e na qualidade da forragem ingerida (Pedroso et al., 2004a; Prache et al., 1997). Sabe-se que, ao longo do período diurno, quando normalmente ocorre 95\% do pastejo diário (Rook et al., 2002), são comuns variações nas necessidades nutricionais, da quantidade e da qualidade da dieta ingerida (Provenza \& Lauchbaungh, 1999; Bergreen-Thomas \& Hohboken, 1986). Entretanto, não há informações de como estas estratégias de pastejo variam ao longo do mesmo período e de quando a

Correspondências devem ser enviadas para: medeiror@orion.ufrgs.br 
pastagem apresenta diferentes níveis de qualidade determinados por variação no estádio fenológico das plantas. No entanto, em virtude da necessidade de manutenção de adequada sanidade e nutrição do rebanho durante todo o ano, é necessária a realização de práticas de manejo, como suplementação, aplicação de vermífugo, entre outras. Esses eventos interferem no ritmo de atividade natural dos animais, tornando fundamental o estudo do padrão diário de variação do ritmo de atividades dos ovinos para redução destas interferências no comportamento natural de pastejo.

Como recurso para gerar informações que possibilitem o manejo mais eficiente do rebanho ovino visando melhorar seus índices de produtividade, realizou-se este estudo com o objetivo de avaliar as estratégias de pastejo no período diurno de ovelhas em final de gestação e início de lactação em pastagem de azevém anual em três diferentes estádios fenológicos.

\section{Material e Métodos}

O experimento foi conduzido no ano 2000, em uma área de 1,3 ha de azevém anual (Lolium multiflorum Lam.) cv. Comum RS em um “Argissolo Vermelho Distrófico Típico", com B textural (EMBRAPA, 1999). Foram utilizadas ovelhas Corriedale em fim de gestação e início de lactação, mantidas em sistema de lotação contínua, com oferta média diária de forragem de $15 \%$ do peso vivo do animal (15 kg de MS/100 kg PV animal/dia), ajustada pela técnica put and take (Soares et al., 2006). A condição qualiquantitativa da pastagem foi determinada considerando sua altura média, obtida com o uso do sward stick (Pontes et al., 2003). A massa de forragem foi estimada pela técnica da dupla amostragem com o uso do disco graduado e as frações de folhas verdes, colmo e material morto, por meio de análise bromatológica e determinação dos teores de PB e da digestibidade in vitro da MO.

As observações comportamentais, realizadas por dois observadores independentes, ocorreram em três períodos de cinco dias consecutivos, representativos dos diferentes estádios fenológicos do azevém anual na pastagem: a) vegetativo (EV - 21 a 25 de julho - 19 ovelhas observadas); b) pré-florescimento (EP - 04 a 08 de outubro - 17 ovelhas observadas); c) florescimento (EF - 07 a 11 de novembro 13 ovelhas observadas), determinados segundo escala proposta por Moore et al. (1991). Para esta verificação, foram retiradas em intervalos semanais 16 amostras compostas de quatro linhas de $30 \mathrm{~cm}$ da pastagem com a finalidade de identificar, nos cinco perfilhos principais, o momento de transição entre os estádios fenológicos. A presença de nós visíveis ou palpáveis permitiu a determinação da passagem do estádio vegetativo para o início do préflorescimento. O momento da passagem entre o EP e o início do EF foi determinado pela combinação entre a porcentagem de perfilhos com nós palpáveis ou visíveis (Moore et al. 1991) e a emissão das primeiras panículas (Tabela 1). Estes dois momentos de transição foram associados a graus-dia de crescimento (GDC), obtidos pela fórmula (GDC $=[$ “(Tmax.+ Tmin $) / 2]-$ Tbase $)$ com Tbase $=$ zero, a partir de dados coletados e armazenados por registradores de temperatura (datalogger tipo HOBO Proseries, Onset Computers) mantidos no interior do dossel.

Do nascer ao pôr-do-sol, foram registrados a cada 10 minutos a freqüência de bocados, peso de bocados, o teor de PB da forragem ingerida, o tempo de pastejo e o consumo de forragem. Para verificação da taxa de bocados, foi adotado o método do tempo de 20 bocados (Jamieson \& Hodgson, 1979), realizando-se, posteriormente, a conversão dos valores obtidos para número de bocados por minuto. O peso de bocado foi determinado de forma simultânea à estimação da freqüência de bocado, por meio de coletas manuais realizadas de forma a reproduzir o peso de bocado de cinco ovelhas com mesmo peso e mesma largura de arcada dentária, escolhidas para este fim. Cada amostra destinada à determinação do peso de bocado foi composta por cinco coletas manuais (hand-plucking), cada uma equivalente a um bocado realizado pelo animal. A calibração da técnica de coleta manual foi feita de forma comparativa, utilizando-se uma ovelha fistulada no esôfago, a partir de uma regressão utilizada para corrigir os valores obtidos nas coletas manuais (Figura 1).

Utilizaram-se amostras coletadas através de fístula esofágica (constituídas pela forragem ingerida em 80 bocados) e por simulação manual de 20 bocados.

Tabela 1 - Porcentagem de nós palpáveis ou visíveis dos perfilhos principais no início dos estádios pré-florescimento e de florescimento e graus-dia de crescimento (GDC) na pastagem de azevém anual

Table 1 - Percentage of palpable or visible nodes in the main tillers at the beginning of the pre-flowering and early-flowering phenological growth stages and growth degree-days (GDD) accumulation in annual ryegrass pastures

\begin{tabular}{|c|c|c|c|}
\hline $\begin{array}{l}\text { Transição de estádios } \\
\text { Transition stages }\end{array}$ & $\begin{array}{l}\text { Data } \\
\text { Date }\end{array}$ & $\begin{array}{l}\text { Nós palpáveis }(\%) \\
\text { Palpable nodes }(\%)\end{array}$ & $\begin{array}{c}\mathrm{GDC}\left({ }^{\circ} \mathrm{C}\right) \\
G D D\left({ }^{\circ} \mathrm{C}\right)\end{array}$ \\
\hline Pré-florescimento & $23 / 09$ & 75 & 2144 \\
\hline $\begin{array}{l}\text { Pre-flowering } \\
\text { Florescimento }^{1}\end{array}$ & $08 / 10$ & 90 & 2422 \\
\hline Early-flowering & & & \\
\hline
\end{tabular}

1 Primeiras panículas visíveis

1 First visible panicles. 


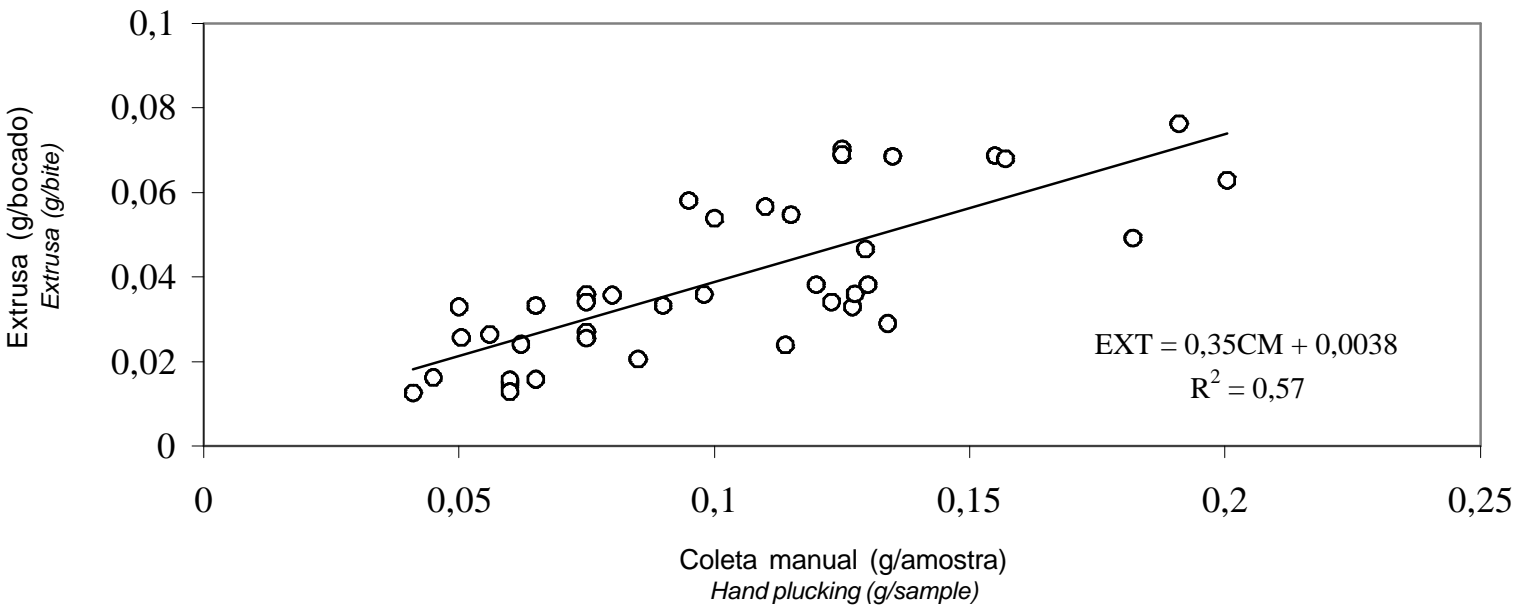

Figura 1 - Relação entre peso seco de bocado estimado por coleta de extrusa (EXT) do animal fistulado e o peso seco da amostra obtida por coleta manual (CM).

Figure 1 - Relationship between bite dry weight estimated by extrusa sample of fistulated animal and the sample dry weight obtained by hand plucking

Posteriormente, as amostras obtidas pela coleta manual serviram também para determinação do teor de PB da forragem aparentemente ingerida.

O tempo de pastejo foi calculado a partir de registros da porcentagem de animais nesta atividade a cada 10 minutos, considerando todas as ovelhas do lote, pela seguinte fórmula:

$\mathrm{TP} 10 \min =10 *(\%$ pastejo $1+\%$ pastejo 2$) / 200$, em que: $\%$ pastejo $1=\%$ de animais pastejando em determinado horário; $\%$ pastejo $2=\%$ de animais pastejando no horário imediatamente posterior.

Desta forma, o tempo total de pastejo diurno correspondeu ao somatório dos valores calculados para cada intervalo de dez minutos.

O critério de observar todas as ovelhas do lote foi adotado pela maior praticidade encontrada pelos observadores de identificar determinada atividade em maiores distâncias sem a identificação individual dos animais, pois possuíam peso e condição corporal semelhantes, pertenciam à mesma raça e estavam em mesma fase fisiológica.

O consumo de forragem foi obtido pela seguinte fórmula: Consumo $=\mathrm{TP} \times \mathrm{FB} \times \mathrm{PB}$, em que: $\mathrm{TP}=$ tempo de pastejo; em que $\mathrm{FB}=$ freqüência de bocados; e $\mathrm{PB}=$ peso de bocados.

Os dados foram submetidos à análise de variância, em um delineamento inteiramente casualizado, em que as repetições consistiram dos cinco dias de observações do nascer ao pôr-do-sol, realizadas em cada estádio, EV, EP e EF (Pedroso et al., 2004a). Os tratamentos consistiram dos diferentes estádios fenológicos, de modo que, para a verificação do detalhamento do comportamento diurno, utilizou-se cada horário previamente definido como fator.
Para as avaliações na pastagem, retiraram-se 26 amostras, marcadas por transectas atravessando diagonalmente o local, de modo a descrever um percurso em "W", visando à melhor representatividade da área. As amostras foram testadas quanto à significância da auto-correlação espacial e, na ausência de significância, foram consideradas repetições verdadeiras (Quadros \& Bandinelli, 2005). Em todos os casos, as médias foram comparadas pelo teste de DMS com nível mínimo de 5\% de significância.

\section{Resultados e Discussão}

A pastagem de azevém anual, no decorrer dos três estádios fenológicos, manteve-se com oferta média de folhas verdes superior a 5\% do PV. Entretanto, em virtude da diminuição da participação de folhas na pastagem, com o avançar do ciclo de crescimento, a altura e a massa de forragem aumentaram para manutenção das ofertas pretendidas (Tabela 2). Desse modo, os animais apresentaram diferentes respostas comportamentais para cada estádio fenológico das plantas na pastagem.

No estádio vegetativo, não foram verificadas variações significativas durante o período diurno para taxa de bocado, peso de bocado e teor de PB da forragem ingerida (Tabela 3). A alta participação de folhas verdes nesta fase provavelmente determinou valores máximos para estas variáveis comportamentais do nascer ao pôr-do-sol. Por outro lado, o tempo de pastejo variou com o avanço dos horários diurnos, resultando em variações no consumo de MS e PB pelos animais.

Os maiores valores $(\mathrm{P}<0,05)$ para tempo de pastejo e consumos de MS e PB foram verificados nos horários de 7 
Tabela 2 - Altura média do dossel (AMD), oferta de folhas verdes (OFV) e massas de forragem (MF), de folha verde (FV) e de material morto (MM) da pastagem de azevém anual em três estádios fenológicos

Table 2 - $\quad$ Average sward height (ASH), green leaf on offer (GLO), yield of herbage mass (Mass), and proportion of green leaf (GL), stem and dead herbage $(D H)$ of annual ryegrass pasture at three different phenological growth stages

\begin{tabular}{|c|c|c|c|c|c|c|}
\hline Estádio fenológico & AMD $(\mathrm{cm})$ & OFV (PV\%) & MF (kg MS/ha) & MFV (\%) & Colmo $(\%)$ & $\mathrm{MM}(\%)$ \\
\hline Phenological stage & $A S H(\mathrm{~cm})$ & $G L O(\% B W)$ & Mass $(\mathrm{kg} D M / \mathrm{ha})$ & $G L(\%)$ & Stem (\%) & $D H(\%)$ \\
\hline Vegetativo (Vegetative) & $8,57 \mathrm{a}$ & $9,6 \mathrm{a}$ & $1610 \mathrm{a}$ & $68 \mathrm{a}$ & $18 \mathrm{a}$ & $13 \mathrm{a}$ \\
\hline Pré-florescimento (Pre-flowering) & $9,86 \mathrm{a}$ & $6,56 \mathrm{a}$ & $2235 b$ & $48 \mathrm{~b}$ & $34 \mathrm{~b}$ & $18 \mathrm{~b}$ \\
\hline Florescimento (Flowering) & $16,11 \mathrm{~b}$ & $5,46 \mathrm{a}$ & $3408 \mathrm{c}$ & $22 \mathrm{c}$ & $38 \mathrm{~b}$ & $40 \mathrm{c}$ \\
\hline
\end{tabular}

Valores com letras iguais, na mesma coluna, não diferem pelo teste DMS a $5 \%$ de significância.

Values followed by the same letter within a column are not significantly different by LSD test at 0.05 probability level.

Tabela 3 - Tempo de pastejo, taxa, peso e teor de PB do bocado e consumos de MS e PB por ovelhas durante o estádio vegetativo em diferentes horários do período diurno no período de 21 a 25 de julho de 2000

Table 3 - Diurnal grazing time, bite rate, bite weight, CP/bite, and intakes of DM and CP by ewes grazing annual ryegrass pasture at the vegetative phenological growth stage (from July 21 to 25 of 2000)

\begin{tabular}{|c|c|c|c|c|c|c|}
\hline \multirow[t]{2}{*}{$\begin{array}{l}\text { Horário } \\
\text { Time }\end{array}$} & \multicolumn{6}{|c|}{$\begin{array}{c}\text { Estádio vegetativo } \\
\text { Vegetative stage }\end{array}$} \\
\hline & $\begin{array}{c}\text { Tempo de } \\
\text { pastejo }(\mathrm{min}) \\
\text { Grazing time }(\mathrm{min})\end{array}$ & $\begin{array}{l}\text { Taxa de } \\
\text { bocado (boc/min) } \\
\text { Bite rate (bite/min) }\end{array}$ & $\begin{array}{c}\text { Peso de } \\
\text { bocado }(\mathrm{g}) \\
\text { Bite weight }(\mathrm{g})\end{array}$ & $\begin{array}{c}\mathrm{PB}(\%) \\
C P(\%)\end{array}$ & $\begin{array}{c}\text { Consumo de } \\
\text { MS (g/an.) } \\
\text { DM intake (g/animal) }\end{array}$ & $\begin{array}{c}\text { Consumo de } \\
\text { PB (g/an.) } \\
\text { CP intake (g/animal) }\end{array}$ \\
\hline $7 \mathrm{~h}-7 \mathrm{~h} 50$ & $42,9 a$ & $52,51 \mathrm{a}$ & $0,068 \mathrm{a}$ & $24,50 \mathrm{a}$ & $153,28 \mathrm{a}$ & $37,56 \mathrm{a}$ \\
\hline $8 h-8 h 50$ & $12,2 \mathrm{c}$ & $52,19 \mathrm{a}$ & $0,060 \mathrm{a}$ & $24,47 \mathrm{a}$ & $38,20 \mathrm{c}$ & $9,35 \mathrm{c}$ \\
\hline $9 h-9 h 50$ & $12,3 \mathrm{c}$ & $52,11 \mathrm{a}$ & $0,060 \mathrm{a}$ & $24,47 \mathrm{a}$ & $38,56 \mathrm{c}$ & $9,44 \mathrm{c}$ \\
\hline $10 \mathrm{~h}-10 \mathrm{~h} 50$ & $29,2 b$ & $52,72 \mathrm{a}$ & $0,061 \mathrm{a}$ & $24,49 \mathrm{a}$ & $94,44 b$ & $23,13 b$ \\
\hline $11 \mathrm{~h}-11 \mathrm{~h} 50$ & $32,4 b$ & $52,61 \mathrm{a}$ & $0,062 \mathrm{a}$ & $24,49 \mathrm{a}$ & $106,04 b$ & $25,97 b$ \\
\hline $12 \mathrm{~h}-12 \mathrm{~h} 50$ & $24,5 b$ & $52,72 \mathrm{a}$ & $0,061 \mathrm{a}$ & $24,49 \mathrm{a}$ & $79,24 b$ & $19,41 \mathrm{~b}$ \\
\hline $13 \mathrm{~h}-13 \mathrm{~h} 50$ & $28,6 \mathrm{~b}$ & $53,03 \mathrm{a}$ & $0,061 \mathrm{a}$ & $24,49 a$ & $92,73 b$ & $22,71 b$ \\
\hline $14 \mathrm{~h}-14 \mathrm{~h} 50$ & $27,7 b$ & $52,80 a$ & $0,061 \mathrm{a}$ & $24,49 a$ & $89,69 b$ & $21,96 b$ \\
\hline $15 \mathrm{~h}-15 \mathrm{~h} 50$ & $32,4 b$ & $52,83 \mathrm{a}$ & $0,063 \mathrm{a}$ & $24,50 \mathrm{a}$ & $107,97 b$ & $26,45 b$ \\
\hline $16 \mathrm{~h}-16 \mathrm{~h} 50$ & $39,3 \mathrm{a}$ & $52,85 \mathrm{a}$ & $0,065 \mathrm{a}$ & $24,50 \mathrm{a}$ & $134,27 \mathrm{a}$ & $32,90 a$ \\
\hline $17 \mathrm{~h}-18 \mathrm{~h}$ & $40,4 \mathrm{a}$ & $52,65 \mathrm{a}$ & $0,068 \mathrm{a}$ & $24,49 \mathrm{a}$ & $144,06 \mathrm{a}$ & $35,28 \mathrm{a}$ \\
\hline
\end{tabular}

Valores com letras iguais, na mesma coluna, não diferem pelo teste DMS a 5\% de significância.

Values followed by the same letter within a column are not significantly different by LSD test at 0.05 probability level.

às $7 \mathrm{~h} 50$ e de $16 \mathrm{~h}$ às $18 \mathrm{~h}$ e os menores, nos horários de $8 \mathrm{~h}$ às 9h50. Considerando esses resultados, sugere-se que interferências na prática de pastejo para outros fins de manejo sejam feitas nos horários de $8 \mathrm{~h}$ e $9 \mathrm{~h} 50$, quando a atividade de pastejo é menor.

No estádio pré-florescimento, em decorrência do alongamento dos entrenós, a relação folha:colmo foi reduzida em comparação ao EV. Estas mudanças na estrutura e na qualidade da forragem ofertada determinaram mudanças comportamentais ao longo dos horários diurnos. Quando a maior parte do lote estava em pastejo, os animais estavam em intensa competição pelo consumo de forragem (das $7 \mathrm{~h}$ às $7 \mathrm{~h} 50$ e das $17 \mathrm{~h}$ às $19 \mathrm{~h}$ ), verificando-se menor teor de PB na forragem ingerida, aumentos da freqüência de bocados e do peso de bocados e maiores consumos de MS e de PB (Tabela 4).

Essa estratégia reflete a tentativa do animal em garantir a quantidade de forragem necessária ao longo do dia, o que, provavelmente é conseqüência da maior competição pela forragem disponível, decorrente da maior presença de animais em atividade de pastejo em mesmo intervalo de tempo (Armstrong et al., 1995). Contudo, nos horários de menor atividade de pastejo, verificou-se maior intervalo de bocados e menor massa de forragem coletada por bocado, possibilitando a ingestão de frações com maior teor de PB (25,3\%). Esse comportamento é considerado estratégia típica de ruminantes pela qual, depois de alcançada a saciedade física (resultante da estimulação dos receptores volumétricos do rúmen), inicia-se uma nova fase em que o aumento da qualidade da dieta é priorizado (Provenza \& Lauchbaungh, 1999). Todavia, este comportamento refletiu-se em menores consumos de MS e PB em relação aos horários de maior intensidade de pastejo.

Os resultados sugeriram que práticas de manejo como a suplementação alimentar a campo devem ser feitas em horários próximos às 9 h e ao meio-dia. Assim, seriam 
Tabela 4 - Tempo de pastejo, taxa, peso e teor de PB do bocado e consumos de MS e PB por ovinos durante o estádio de pré-florescimento em diferentes horários do período diurno no período de 04 a 08 de outubro de 2000

Table 4 - Diurnal grazing time, bite rate, bite weight, CP/bite, and intakes of DM and CP by ewes grazing annual ryegrass pasture at the pre-flowering phenological growth stage (from October 4 to 8 of 2000)

\begin{tabular}{|c|c|c|c|c|c|c|}
\hline \multirow[t]{2}{*}{$\begin{array}{l}\text { Horário } \\
\text { Time }\end{array}$} & \multicolumn{6}{|c|}{$\begin{array}{c}\text { Estádio pré-florescimento } \\
\text { Pre-flowering stage }\end{array}$} \\
\hline & $\begin{array}{c}\text { Tempo de } \\
\text { pastejo }(\mathrm{min}) \\
\text { Grazing time }(\mathrm{min})\end{array}$ & $\begin{array}{l}\text { Taxa de } \\
\text { bocado (boc/min) } \\
\text { Bite rate (bite/min) }\end{array}$ & $\begin{array}{c}\text { Peso de } \\
\text { bocado }(\mathrm{g}) \\
\text { Bite weight }(g)\end{array}$ & $\begin{array}{c}\mathrm{PB}(\%) \\
C P(\%)\end{array}$ & $\begin{array}{c}\text { Consumo de } \\
\text { MS (g/an.) } \\
\text { DM intake (g/animal) }\end{array}$ & $\begin{array}{c}\text { Consumo de } \\
\text { PB (g/an.) } \\
\text { CP intake (g/animal) }\end{array}$ \\
\hline $7 \mathrm{~h}-7 \mathrm{~h} 50$ & $46,98 \mathrm{a}$ & $55,23 \mathrm{ab}$ & $0,0463 \mathrm{ab}$ & $23,57 \mathrm{bc}$ & $120,15 \mathrm{a}$ & $28,31 \mathrm{a}$ \\
\hline $8 h-8 h 50$ & $15,22 \mathrm{c}$ & $52,04 \mathrm{bc}$ & $0,0382 \mathrm{bc}$ & $25,29 \mathrm{ab}$ & $30,23 c$ & $7,65 \mathrm{c}$ \\
\hline $9 h-9 h 50$ & $23,97 b$ & $52,52 b$ & $0,0396 b$ & $25,10 b$ & $49,87 b$ & $12,52 b$ \\
\hline $10 \mathrm{~h}-10 \mathrm{~h} 50$ & $28,68 b$ & $53,46 b$ & $0,0415 b$ & $24,42 b$ & $63,59 b$ & $15,53 b$ \\
\hline $11 \mathrm{~h}-11 \mathrm{~h} 50$ & $23,23 b$ & $52,90 \mathrm{~b}$ & $0,0405 b$ & $24,90 b$ & $49,77 b$ & $12,39 b$ \\
\hline $12 \mathrm{~h}-12 \mathrm{~h} 50$ & $15,59 \mathrm{c}$ & $51,73 \mathrm{bc}$ & $0,0376 b c$ & $25,51 \mathrm{ab}$ & $30,29 c$ & $7,73 c$ \\
\hline $13 \mathrm{~h}-13 \mathrm{~h} 50$ & $32,59 b$ & $53,64 b$ & $0,0424 b$ & $24,47 b$ & $74,09 \mathrm{~b}$ & $18,13 b$ \\
\hline $14 h-14 h 50$ & $31,23 b$ & $53,52 b$ & $0,0418 \mathrm{~b}$ & $24,41 b$ & $69,83 b$ & $17,04 \mathrm{~b}$ \\
\hline $15 \mathrm{~h}-15 \mathrm{~h} 50$ & $30,59 b$ & $53,34 b$ & $0,0411 b$ & $24,47 b$ & $67,13 b$ & $16,43 b$ \\
\hline $16 \mathrm{~h}-16 \mathrm{~h} 50$ & $35,35 b$ & $54,11 b$ & $0,0433 b$ & $24,12 b$ & $82,86 b$ & $19,99 b$ \\
\hline $17 \mathrm{~h}-17 \mathrm{~h} 50$ & $45,65 \mathrm{a}$ & $55,65 \mathrm{ab}$ & $0,0475 \mathrm{ab}$ & $23,39 \mathrm{bc}$ & $120,75 \mathrm{a}$ & $28,24 \mathrm{a}$ \\
\hline $18 \mathrm{~h}-19 \mathrm{~h}$ & $55,31 \mathrm{a}$ & $56,45 \mathrm{ab}$ & $0,0498 \mathrm{ab}$ & $23,04 \mathrm{bc}$ & $155,50 \mathrm{a}$ & $35,82 \mathrm{a}$ \\
\hline
\end{tabular}

VValores com letras iguais, na mesma coluna, não diferem pelo teste DMS a $5 \%$ de significância.

Values followed by the same letter within a column are not significantly different by LSD test at 0.05 probability level.

respeitados os principais horários de pastejo, em termos quantitativos (início da manhã e final de tarde), e os animais receberiam suplemento alimentar de melhor qualidade justamente nos horários em que são mais seletivos e que consomem menos. Rovira (1997), referindo-se ao comportamento ingestivo de bovinos, não recomendou a suplementação aos animais nas primeiras horas da manhã, pois, em vez de complementar, estar-se-ia substituindo a dieta disponível na pastagem pelo suplemento fornecido.

No estádio de florescimento, no entanto, houve certa uniformização da pastagem com a totalidade dos perfilhos em florescimento. Essa estrutura com elevada participação de colmo (38\%) e material senescente (40\%) provavelmente limitou a ação seletiva dos animais, que, conseqüentemente, apresentaram variações na taxa e no peso de bocados e no teor de PB ingerida durante o período diurno. É possível que o efeito conjunto da condição da pastagem e da elevação da temperatura neste estádio fenológico $\left(\mathrm{EP}-15^{\circ} \mathrm{C} ; \mathrm{EF}-22^{\circ} \mathrm{C}\right)$ tenha estimulado os animais ao pastejo durante o período noturno, na tentativa de satisfazer suas exigências nutricionais diárias, mesmo que não tenham sido alcançadas (Pedroso et al., 2004b). Parte desta explicação também reside no fato de que, nas primeiras horas do dia, registrou-se um curto tempo de atividade de pastejo (até as 8 h50) e de baixos consumos de forragem e PB. Com a provável troca dos períodos de pastejo (preferência pelo pastejo noturno em vez de ao início do período diurno), houve maior necessidade de outras atividades como descanso e ruminação.
No restante do período diurno, o tempo de pastejo e, conseqüentemente, os consumos de MS e PB foram crescentes até o final da tarde (19h30). Estes dados sugerem que o momento mais adequado para intervenções no ritmo natural de pastejo seria nas primeiras horas do dia (até as 8h50), pois, nos primeiros horários matinais (7h às 7h50), a atividade de pastejo é menor.

Entretanto, nos três estádios fenológicos, ao final da tarde, foram verificados os maiores tempos de pastejo e consumos de MS e PB do período diurno. Vários autores registraram esses elevados valores momentos antes do anoitecer (Barbosa et al., 2004; Orr et al.,1997) e atribuíram o fato à composição química das plantas, que apresentam os maiores teores de MS e carboidratos nestes horários. Parsons et al. (1994) acrescentaram que a maior ingestão de forragem ao final da tarde ocorre porque os animais dispõem do período noturno para a atividade de ruminação.

Como resultado destas evoluções, foram verificados maiores consumos de MS e PB para EV e EP (Tabela 6). O consumo de $\mathrm{PB}$ foi muito próximo do recomendado para mantença de uma ovelha lactante com ganho médio diário de $25 \mathrm{~g}$ (240 g/dia). Todavia, o consumo de MS de 1.740 g/dia (Ganzábal, 1997), exigido por ovelhas em lactação, foi suprido em $62 \%$.

No estádio de florescimento, tanto o consumo diurno de MS como o de PB, foram considerados insatisfatórios para mantença destes animais de elevadas exigências nutricionais. De fato, o desempenho animal neste trabalho revelou uma importante perda de peso (152 g/dia) destes animais. Segundo Pedroso et al. (2004b), sob baixa 
Tabela 5 - Tempo de pastejo, taxa, peso e teor de PB do bocado e consumos de MS e PB por ovinos durante o estádio de florescimento em diferentes horários do período diurno

Table 5 - $\quad$ Diurnal grazing time, bite rate, bite weight, CP/bite, and intakes of DM and CP by ewes grazing annual ryegrass pasture at the flowering phenological growth stage

\begin{tabular}{|c|c|c|c|c|c|c|}
\hline \multirow[t]{2}{*}{$\begin{array}{l}\text { Horário } \\
\text { Time }\end{array}$} & \multicolumn{6}{|c|}{$\begin{array}{c}\text { Estádio florescimento } \\
\text { Flowering stage }\end{array}$} \\
\hline & $\begin{array}{c}\text { Tempo de } \\
\text { pastejo }(\mathrm{min}) \\
\text { Grazing time }(\mathrm{min})\end{array}$ & $\begin{array}{l}\text { Taxa de } \\
\text { bocado (boc/min) } \\
\text { Bite rate (bite/min) }\end{array}$ & $\begin{array}{c}\text { Peso de } \\
\text { bocado }(\mathrm{g}) \\
\text { Bite weight }(g)\end{array}$ & $\begin{array}{l}\mathrm{PB}(\%) \\
C P(\%)\end{array}$ & $\begin{array}{c}\text { Consumo de } \\
\text { MS (g/an.) } \\
\text { DM intake (g/animal) }\end{array}$ & $\begin{array}{c}\text { Consumo de } \\
\text { PB (g/an.) } \\
\text { CP intake (g/animal) }\end{array}$ \\
\hline $7 h-7 h 50$ & $2,21 \mathrm{c}$ & $39,89 a$ & $0,049 \mathrm{a}$ & $14,34 \mathrm{a}$ & $4,32 \mathrm{c}$ & $0,62 \mathrm{c}$ \\
\hline $8 h-8 h 50$ & $5,87 \mathrm{c}$ & $39,89 a$ & $0,049 \mathrm{a}$ & $14,34 \mathrm{a}$ & $11,46 \mathrm{c}$ & $1,64 \mathrm{c}$ \\
\hline $9 h-9 h 50$ & $19,52 b$ & $40,20 \mathrm{a}$ & $0,049 \mathrm{a}$ & $14,51 \mathrm{a}$ & $38,12 b$ & $5,53 b$ \\
\hline $10 \mathrm{~h}-10 \mathrm{~h} 50$ & $25,29 b$ & $40,15 \mathrm{a}$ & $0,047 \mathrm{a}$ & $14,95 \mathrm{a}$ & $48,14 b$ & $7,20 \mathrm{~b}$ \\
\hline $11 \mathrm{~h}-11 \mathrm{~h} 50$ & $24,33 b$ & $39,98 \mathrm{a}$ & $0,047 \mathrm{a}$ & $15,00 \mathrm{a}$ & $45,99 b$ & $6,90 \mathrm{~b}$ \\
\hline $12 \mathrm{~h}-12 \mathrm{~h} 50$ & $24,42 b$ & $39,87 \mathrm{a}$ & $0,048 \mathrm{a}$ & $14,66 \mathrm{a}$ & $46,91 b$ & $6,88 b$ \\
\hline $13 \mathrm{~h}-13 \mathrm{~h} 50$ & $25,77 b$ & $40,49 a$ & $0,048 \mathrm{a}$ & $14,80 \mathrm{a}$ & $49,91 b$ & $7,39 \mathrm{~b}$ \\
\hline $14 \mathrm{~h}-14 \mathrm{~h} 50$ & $24,52 b$ & $40,17 \mathrm{a}$ & $0,048 \mathrm{a}$ & $14,76 \mathrm{a}$ & $47,19 b$ & $6,97 b$ \\
\hline $15 \mathrm{~h}-15 \mathrm{~h} 50$ & $20,10 b$ & $39,85 \mathrm{a}$ & $0,048 \mathrm{a}$ & $14,72 \mathrm{a}$ & $38,44 b$ & $5,66 \mathrm{~b}$ \\
\hline $16 \mathrm{~h}-16 \mathrm{~h} 50$ & $34,36 a$ & $40,80 \mathrm{a}$ & $0,047 \mathrm{a}$ & $14,97 \mathrm{a}$ & $66,46 a$ & $9,95 \mathrm{a}$ \\
\hline $17 \mathrm{~h}-17 \mathrm{~h} 50$ & $43,04 \mathrm{a}$ & $42,09 a$ & $0,047 \mathrm{a}$ & $15,06 \mathrm{a}$ & $85,58 \mathrm{a}$ & $12,89 \mathrm{a}$ \\
\hline $18 \mathrm{~h}-19 \mathrm{~h}$ & $31,99 \mathrm{a}$ & $40,61 \mathrm{a}$ & $0,047 \mathrm{a}$ & $15,20 \mathrm{a}$ & $60,78 \mathrm{a}$ & $9,24 \mathrm{a}$ \\
\hline $19 h-19 h 30$ & $33,40 \mathrm{a}$ & $42,48 \mathrm{a}$ & $0,047 \mathrm{a}$ & $15,14 \mathrm{a}$ & $66,77 \mathrm{a}$ & $10,11 \mathrm{a}$ \\
\hline
\end{tabular}

Valores com letras iguais na mesma coluna não diferem pelo teste DMS a $5 \%$ de significância.

Values within a column followed by the same letter are not significantly different by LSD test at 0.05 probability level.

Tabela 6 - Tempo de pastejo, consumo de MS, consumo de MS em \% do PV e consumo de PB por ovinos em pastagem de azevém anual em diferentes estádios fenológicos

Table 6 - Grazing time, DM intake, DM intake in \% of BW, CP intake by sheep on different phenological stages in annual ryegrass pasture

\begin{tabular}{|c|c|c|c|c|}
\hline $\begin{array}{l}\text { Estádio fenológico } \\
\text { Phenological stage }\end{array}$ & $\begin{array}{c}\text { Tempo de pastejo(min/dia) } \\
\text { Grazing time (min/day) }\end{array}$ & $\begin{array}{c}\text { Consumo de } \mathrm{MS}(\mathrm{g} / \mathrm{dia}) \\
\text { DM intake (g/day) }\end{array}$ & $\begin{array}{c}\text { Consumo de MS (\% PV) } \\
\text { DM intake }(\% B W)\end{array}$ & $\begin{array}{c}\text { Consumo de } \mathrm{PB} \text { (g/dia) } \\
C P \text { intake (g/day) }\end{array}$ \\
\hline Vegetativo (Vegetative) & $321,66 a$ & $1.078,01 \mathrm{a}$ & $2,7 \mathrm{a}$ & $264,06 a$ \\
\hline Pré-florescimento (Pre-flowering) & $432,98 b$ & $917,824 \mathrm{a}$ & $2,29 \mathrm{a}$ & $219,40 \mathrm{a}$ \\
\hline Florescimento (Flowering) & $425,96 b$ & $610,32 b$ & $1,52 b$ & $90,83 b$ \\
\hline
\end{tabular}

Valores com letras iguais na mesma coluna não diferem pelo teste DMS a $5 \%$ de significância.

Values within a column followed by the same letter are not significantly different by LSD test at 0.05 level.

qualidade, as variações de tempo de pastejo e no tamanho do bocado não permitem aos animais superar a dificuldade imposta pela condição da pastagem em florescimento para colher uma dieta que atenda às suas exigências nutricionais para mantença e aleitamento.

\section{Conclusões}

Os maiores tempos de pastejo e consumos de MS e PB ocorrem no final do período diurno para os estádios vegetativo, pré-florescimento e de florescimento.

Nos dois estádios iniciais, estes maiores valores também ocorrem nos primeiros momentos de luz natural.

No pré-florescimento, em alta atividade de pastejo, as ovelhas elevam a taxa de bocados e a quantidade de forragem coletada por bocado e diminuem a qualidade do ingerido. No estádio de florescimento, reduzem os tempos de pastejo e o consumo de MS e de PB ao longo de todo o período diurno, como conseqüência da baixa qualidade da forragem disponível e da estrutura da vegetação.

\section{Literatura Citada}

ARMSTRONG, R.H.; ROBERTSON, E.; HUNTER, E.A. The effect of sward height and its direction of change on the herbage intake, diet selection and performance of weaned lambs grazing ryegrass swards. Grass and Forage Science, v.50, p.389-398, 1995.

BARbosa, C.M.P.; CARVAlho, P.C.F.; SILVA, M.A. et al. Comportamento ingestivo diurno de cordeiros em pastagem de azevém anual. In: REUNIÃO ANUAL DA SOCIEDADE BRASILEIRA DE ZOOTECNIA, 41., 2004, Recife. Anais... Recife: Sociedade Brasileira de Zootecnia, 2004. (CD-ROM).

BERGGREN-THOMAS, B.; HOHENBOKEN, W.D. The effects of size-breed, forage availability and weather on the grazing behaviour of crossbred ewes. Applied Animal Behavior Science, v.15, p.217-228, 1986.

EMPRESA BRASILEIRA DE PESQUISA E AGROPECUÁRIA EMBRAPA. Sistema Brasileiro de Classificação de Solo. Brasília: Embrapa Cerrados, Rio de Janeiro: Centro Nacional de Pesquisa de Solos, 1999. 412p. 
GANZÁBAL, A. Alimentación de ovinos com pasturas sembradas. Montevideo: Instituto Nacional de Investigación Agropecuaria, 1997. v.84, 44p.

JAMIESON, W.S.; HODGSON, J. The effect of daily herbage allowance and sward characteristics upon the ingestive behaviour and herbage intake of calves under strip-grazing for grazing dairy cows. Grass and Forage Science, v.34, p.69-77, 1979.

MOORE, K.J.; MOSER, L.E.; VOGEL, K.P. et al. Describing and quantifying growth stages of perennial forage grasses. Agronomy Journal, v.83, p.1073-1077, 1991.

ORR, R.J.; PENNING, P.D.; HARVEY, A.; CHAMPION, R.A. Diurnal patterns of intake rate by sheep grazing monocultures of ryegrass or white clover. Applied Animal Behavior Science, v.52, p.65-77, 1997

PARSONS, A.J.; NEWMAN, J.A.; PENNING, P.D. et al. Diet preference of sheep: effects of recent diet, physiological state and species abundance. Journal of Animal Ecology, v.63, p.465-478, 1994 .

PEDROSO, C.E.S.; MEDEIROS, R.B.; ABREU DA SILVA, M. et al. Comportamento de ovinos em gestação e lactação sob pastejo em diferentes estádios fenológicos de azevém anual. Revista Brasileira de Zootecnia, v.33, n.5, p.1340-1344, 2004a.

PEDROSO, C.E.S.; MEDEIROS, R.B.; ABREU DA SILVA, M. et al. Produção de ovinos em gestação e lactação sob pastejo em diferentes estádios fenológicos de azevém anual. Revista Brasileira de Zootecnia, v.33, n.5, p.1345-1350, 2004b.

PONTES, L.S.; NABINGER, C.; CARVALHO, P.C.F. et al. Variáveis morfogênicas e estruturais de azevém anual (Lolium multiflorum Lam.) manejado em diferentes alturas. Revista Brasileira de Zootecnia, v.32, n.4, p.814-820, 2003.
PRACHE, S. Intake rate, intake per bite and time per bite of lactating ewes on vegetative and reproductive swards. Applied Animal Behavior Science, v.52, p.53-64, 1997.

PROVENZA, F.D.; LAUCHBAUNGH, K.L. Foraging on the edge of chaos. In: LAUCHBAUNGH, K.L.; MOSLEY, J.C.; SANDERS, K.D. (Eds.). Grazing behavior of livestock and wildlife. Moscow: University of Idaho, 1999. p.1-12.

QUADROS, F.L.F.; BANDINELLI, D.G. Efeitos da adubação nitrogenada e de sistemas de manejo sobre a morfogênese de Lolium multiflorum Lam. e Paspalum urvillei Steud. em ambiente de várzea. Revista Brasileira de Zootecnia, v.34, n.1, p.44-53, 2005.

ROOK, A.J.; HARVEY, A.; PARSONS, A.J. et al. Effect of long term changes in relative resource availability on dietary preference of grazing sheep for perennial ryegrass and white clover. Grass and Forage Science, v.57, p.54-60, 2002.

ROVIRA, J. Manejo nutritivo de los rodeos de cria en pastoreo. Montevideo: Hemisferio Sur, 1997. 288p.

SOARES, A.B.; MEZZALIRA, J.C.; BUENO, E.A.C. et al. Efeitos de diferentes intensidades de pastejo em pastagem nativa melhorada sobre o desempenho animal. Revista Brasileira de Zootecnia, v.35, n.1, p.75-83, 2006.

THOMAS, B.B.; HOHENBOKEN, W.D. The effects of size-breed, forage availability and weather on the grazing behaviour of crossbred ewes. Applied Animal Behavior Science, v.15, p.217-228, 1986. 